\title{
O papel da mobilidade urbana no fomento à cidade inteligente: 0 caso do Vale do Pinhão, Curitiba/PR 1
}

\section{The urban mobility role in the promotion of a smart city: the case of Vale do Pinhão, Curitiba/PR}

\author{
Witiuk, Rafael1; Skwarok, Aldrei2; Von Der Osten, Fabiana ${ }^{3}$ \\ 1 Pontifícia Universidade Católica do Paraná, Rua Imaculada Conceição, \\ 1155 Prado Velho, Curitiba-PR, Brasil e rafael.witiuk@pucpr.br \\ 2 Pontifícia Universidade Católica do Paraná, aldrei.skwarok@pucpr.br \\ 3 Pontifícia Universidade Católica do Paraná, fabiana.osten@pucpr.br
}

\begin{abstract}
RESUMO
O presente estudo, em um primeiro momento, descreve os conceitos que envolvem o processo de desenvolvimento de espaços inteligentes, sua importância no capitalismo contemporâneo e seu objeto de estudo: o Vale do Pinhão. Foi realizada a revisão da bibliografia existente acerca dos temas mobilidade urbana, cidade inteligente e economia criativa, com o objetivo de apresentar a correlação entre tais temáticas e demonstrar o papel central da mobilidade no âmbito do Projeto Vale do Pinhão e na busca de Curitiba pelo status de cidade inteligente. Como resultado destacam-se as iniciativas relacionadas à promoção da mobilidade ativa e as propostas de mobilidade inteligente, notadamente o uso de tecnologias de transporte inteligente - ITS, em contraponto à elevada participação do veículo automotor individual - que representa $46 \%$ dos deslocamentos na cidade. Conclui-se que este último, é um fator a ser combatido para o fomento da economia criativa sendo necessário ofertar serviços de mobilidade que permitam melhor relação usuário-cidade e melhor condição de infraestrutura, segurança e integração dos meios de transporte.
\end{abstract}

Palavras-chave: Mobilidade urbana, cidade inteligente, economia criativa.

\begin{abstract}
This study presents, firstly, the concepts involving the process of intelligent spaces development, its importance for the current stage of contemporary capitalism and its object of study: the Vale do Pinhão. A literature review was made on urban mobility, smart city and creative economy, to present the correlation between these themes and to demonstrate the central role of mobility in the Vale do Pinhão Project as well as providing to Curitiba a smart city status. As a result, active mobility related initiatives are highlighted, as well as proposals of smart mobility, especially the use of intelligent transport system - ITS, as opposed to the high participation of cars, which represents $46 \%$ of the modal split in the city. In conclusion, the elevated rate of cars participation is a factor to be combated for the promotion of a creative economy and it is necessary to offer mobility services that improves the relation between the

\footnotetext{
${ }^{1}$ WITIUK, Rafael; SKWAROK, Aldrei; VON DER OSTEN, Fabiana. O papel da mobilidade urbana no fomento à cidade inteligente: o caso do vale do pinhão, Curitiba/PR. In: II SIMPÓSIO NACIONAL DE GESTÃO E ENGENHARIA URBANA: SINGEURB, 2019, São Paulo. Anais... Porto Alegre: ANTAC, 2019.
} 
city and their inhabitants and also promotes a better condition of infrastructure, security and integration in transports.

Keywords: Urban mobility, smart city, creative economy.

\section{INTRODUÇÃO}

Atualmente, grande parte das cidades brasileiras têm experimentado níveis de desenvolvimento econômico pouco atrativos dentro das expectativas do atual estágio de evolução do capitalismo moderno, o chamado capitalismo cognitivo (Procopiuck e Freder, 2013). Tendo em vista a aparente decadência do modelo tradicional, a economia criativa tem se apresentado como uma alternativa propícia no enfrentamento dos desafios contemporâneos do desenvolvimento socioeconômico nas cidades (Miguez, 2007). É nessa frente que lideranças municipais e agentes econômicos têm vislumbrado a possibilidade de alavancar a inovação e o crescimento de cidades com o lançamento de projetos como o Vale do Pinhão em Curitiba (Vale do Pinhão, 2017), o Sapiens Park em Florianópolis (Lara et al., 2013), o Porto Digital no Recife (Trindade, 2014), dentre outras iniciativas.

No que se refere à experiência da capital paranaense, a criação do projeto Vale do Pinhão em 2017 (ver Figura 1) é uma iniciativa do município para a revitalização do bairro Rebouças, transformando-o em um espaço inteligente, mais humano, criativo e, principalmente, competitivo em sua capacidade de atrair pessoas e investimentos (Balula, 2010; Vale do Pinhão, 2017).

Figura 1 - Situação do Vale do pinhão local e metropolitana

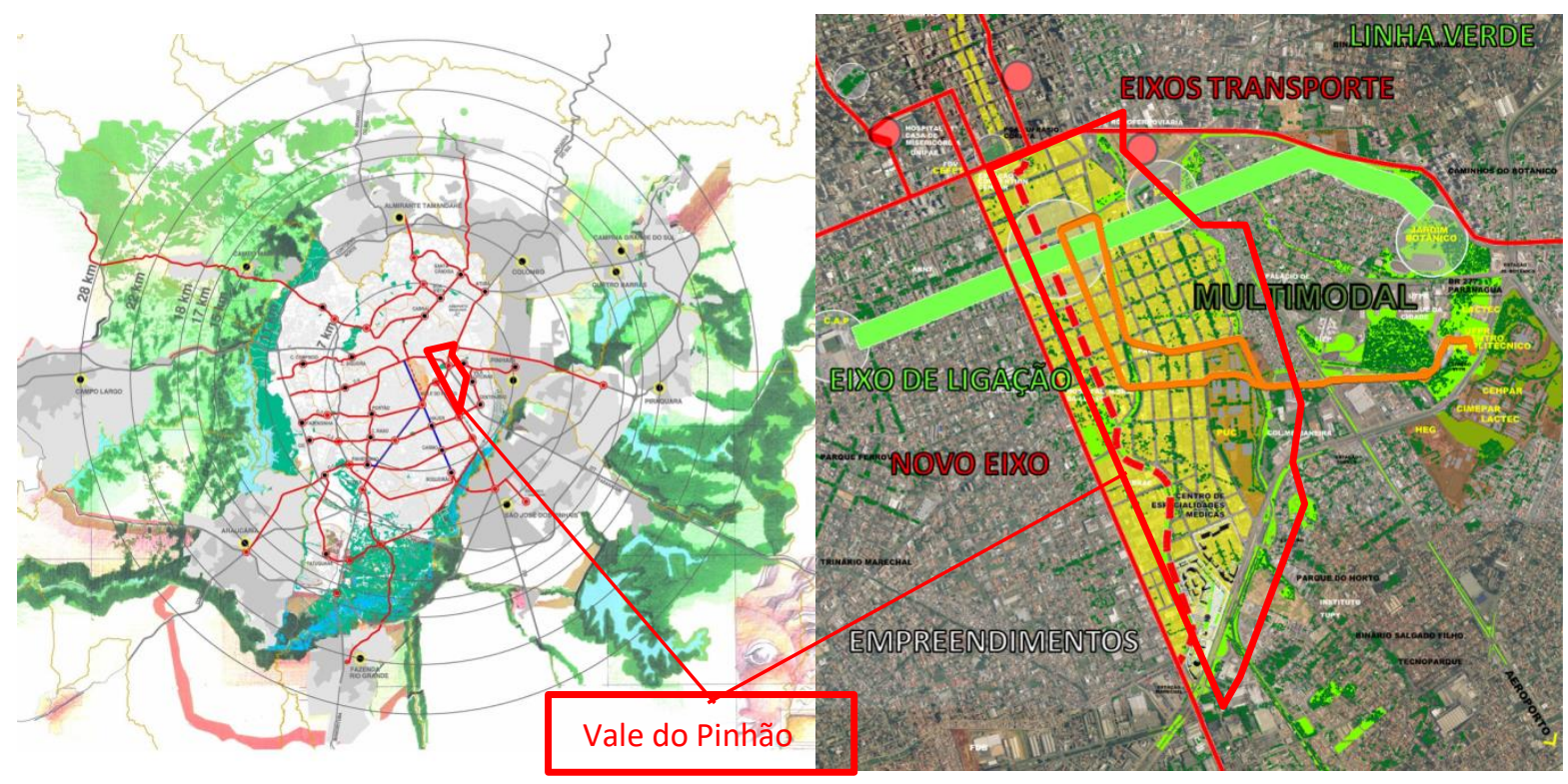

Fonte: Vale do Pinhão (2017)

Segundo Costa et al. (2009), para a estruturação de espaços urbanos inteligentes, é necessário garantir condições adequadas de qualidade de vida e infraestrutura, promovendo a atratividade destas regiões. Para o autor, os fatores preponderantes para que sejam atingidos esses objetivos envolvem a estruturação de uma boa mobilidade urbana. Caragliu et al. (2009) corroboram essa afirmação ao ressaltar o papel da mobilidade urbana e dos transportes como um aspecto importante para a viabilização dos espaços criativos, haja vista sua capacidade de geração de bem-estar. Benevolo et al. (2016) vai além ao atribuir à mobilidade urbana um papel crucial e transversal para o êxito da cidade inteligente.

Tendo em vista a aparente vocação da mobilidade urbana como importante fator na promoção de espaços urbanos criativos, este trabalho tem por objetivo referendar a discussão voltada à criação do Vale do Pinhão, destacando as propostas de mobilidade apresentadas pelo projeto e sua relação com os princípios de desenvolvimento baseados na 
economia criativa. Ainda, busca-se elencar novas propostas de mobilidade visando a consolidação do referido ecossistema criativo, com base em outras experiências descritas na bibliografia.

\section{METODOLOGIA}

No presente estudo foi realizada uma revisão da bibliografia existente acerca do tema, mais precisamente no que tange o projeto Vale do Pinhão, de modo a avaliar a relação entre mobilidade e cidade inteligente em quatro perspectivas: como infraestrutura de suporte ao setor criativo, como ferramenta para revitalização do bairro Rebouças, como fator gerador de atratividade e segundo o conceito de mobilidade inteligente.

\section{MOBILIDADE URBANA E A CRIAÇÃO DE ESPAÇOS CRIATIVOS}

A seguir são apresentadas as quatro perspectivas de análise acerca da mobilidade urbana, o desenvolvimento da economia criativa e a promoção de espaços urbanos inteligentes.

\subsection{A mobilidade urbana como suporte ao setor criativo}

As infraestruturas de transporte e o planejamento da mobilidade urbana dos municípios não apenas viabilizam o processo produtivo e a prestação de serviço, mas garantem as condições para o desenvolvimento do setor criativo apresentadas por Costa et al. (2009), como a multifuncionalidade urbana, a proximidade urbana, a participação cívica e a interculturalidade - na perspectiva do direito de acesso à cidade.

No que se refere à infraestrutura, Giffinger et al. (2010) ressalta ainda o papel da acessibilidade dos espaços, da acessibilidade internacional e da segurança dos sistemas de transporte na classificação de uma cidade inteligente. Em se tratando de infraestrutura, a promoção da acessibilidade vai ao encontro das condições definidas por Costa et al. (2009) e tem sua importância reafirmada por Nascimento et al. (2015) para o desenvolvimento de agrupamentos de empresas do setor criativo. O bairro Rebouças, na escala de Curitiba, tem como principal objetivo concentrar as iniciativas da classe criativa, formando um cluster criativo na cidade por meio da oferta de acessibilidade e infraestrutura.

Em se tratando de acessibilidade internacional, este conceito dialoga com a noção de atratividade exercida pela cidade e a possibilidade de acessá-la seja por transporte aéreo ou terrestre. Em termos de acesso rodoviário, a cidade apresenta um denso rosário de transporte com saídas em diversas direções como pode ser visto na Figura 2 (Huertas, 2016). O desempenho da cidade é igualmente exemplar em se tratando de conectividade aérea, haja vista os elevados índices de satisfação obtidos pelo seu aeroporto (Ministério dos Transportes, Portos e Aviação Civil, 2018).

Quanto a segurança do sistema de transporte, que, em última instância, está sujeita ao grau de dependência e incentivo ao uso do veículo automotor individual, Curitiba apresenta um baixo desempenho devido a participação de $46 \%$ deste meio de transporte na repartição modal da cidade (IPPUC, 2017).

\subsection{A mobilidade urbana como parte integrante da revitalização do bairro Rebouças}

O bairro Rebouças, núcleo do Vale do Pinhão, é o lugar de prototipação onde será desenvolvida uma grande diversidade de propostas relacionadas ao fomento da economia criativa. Da mesma maneira, nessa região serão desenvolvidos projetos de acessibilidade, paisagismo, mobilidade e conectividade da área, como as propostas de revitalização dos espaços e das vias do bairro (Vale do Pinhão, 2017). Segundo Nunes (2017), a revitalização do Rebouças representa uma oportunidade para que sejam reestruturadas as relações do bairro com o resto da aglomeração urbana, tornando-o a porta de entrada da cidade para sua região metropolitana. 
A proposta prevê a reestruturação da oferta de transporte público metropolitano através de quatro ligações intermunicipais que orientam o fluxo de pessoas para a região do Vale do Pinhão conforme apresentado na Figura 1 (IPPUC apud Nunes, 2017). A proposta concede facilidade de acesso ao bairro que, segundo os preceitos de Giffinger e Gudrun (2010), contribui positivamente para criação de um espaço urbano inteligente. No campo da mobilidade ativa, são previstas propostas relacionadas a melhoria da "caminhabilidade" do bairro defendida por Speck (2012) e o favorecimento do uso de modais ativos em geral.

Figura 2 - Configuração territorial do eixo paranaense

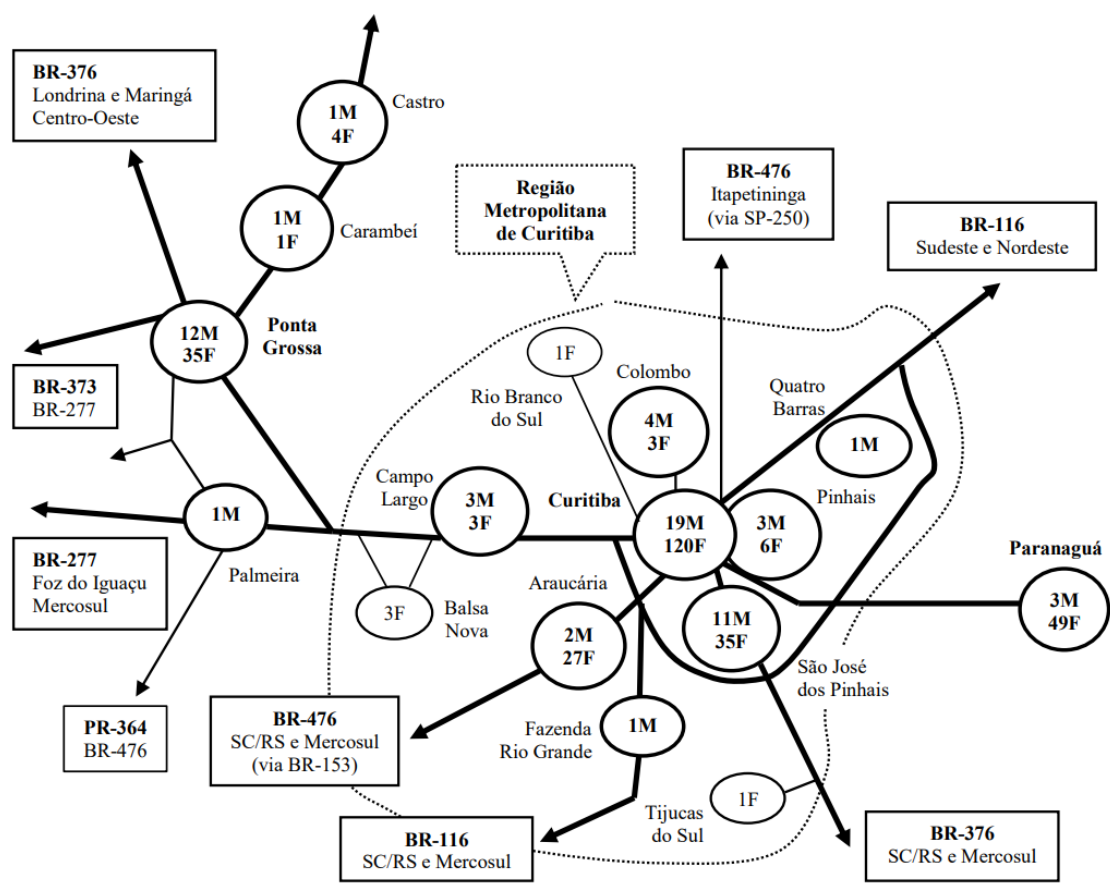

Fonte: Huertas (2016)

\subsection{A Mobilidade urbana como fator gerador de atratividade}

Na prática, uma cidade criativa depende de sua capacidade de atrair gente com habilidades e talentos que contribuam para o desenvolvimento do setor criativo. Nesse sentido, a atratividade representa um fator crucial para o êxito de um ecossistema criativo ao reunir talentos, favorecer a interculturalidade e constituir grande diversidade de procedência de seus habitantes (Ferreira, 2017; Costa et al., 2009).

Em sua história, principalmente ao longo dos anos de 1980 e 1990, Curitiba experimentou um período de grande atratividade em decorrência de sua projeção como um modelo de planejamento urbano, desenvolvimento sustentável e transporte público. Nesse mesmo período, a cidade se tornou a mais populosa do Sul do país.

Além da capital paranaense, o caso da cidade de Portland, nos Estados Unidos, também é considerado um exemplo de como o planejamento urbano e transporte público de qualidade podem promover a atratividade e gerar um ambiente criativo. No início dos anos 1970, período do auge do espraiamento das cidades norte-americanas e da popularização do automóvel, Portland instituiu diretrizes de uso e ocupação do solo associadas a oferta de um sistema de transporte público de qualidade, controlando seu crescimento por setores. Este processo rendeu a Portland a fama de cidade densa, inovadora e planejada (Abbott, 2001), fomentando um intenso crescimento e desenvolvimento da cidade no cenário estadunidense atraindo jovens e indústrias do setor criativo.

Estes dois exemplos demonstram a atratividade exercida por cidades planejadas no cenário nacional e internacional, com melhores índices de mobilidade e acessibilidade. 


\subsection{A Mobilidade urbana inteligente}

Apesar de não ser um requisito para a estruturação da cidade inteligente, as iniciativas discutidas no âmbito da mobilidade inteligente podem ser consideradas cruciais, permeando diversas temáticas envolvendo a sua viabilidade (Benevolo et al., 2016; Costa et al., 2012; Giffinger e Gudrun, 2010).

Com exceção de algumas propostas relacionadas ao uso de combustíveis alternativos e ônibus elétricos no sistema de transporte público, Curitiba tem apresentado parcas soluções em mobilidade inteligente. No Quadro 1 estão dispostas algumas proposições relevantes para o projeto Vale do Pinhão. As propostas estão classificadas segundo três critérios: quanto à sua natureza de uso, podendo ser pública, privada ou ser aplicada à infraestrutura e quanto ao benefício gerado pela sua implementação, que pode ser a redução dos congestionamentos (RC), a redução da polvição do ar (RP), a melhoria da segurança viária (MS), a redução da polvição sonora (RS) e a diminuição dos custos de transporte (RCT).

Quadro 1 - Relação das propostas de mobilidade inteligente e suas características

\begin{tabular}{|c|c|c|c|}
\hline Propostas & Natureza & Benefício & Referência \\
\hline Veículos Elétricos & $\begin{array}{c}\text { Pública / } \\
\text { Privada }\end{array}$ & RPA e RPS & $\begin{array}{c}\text { Benevolo et al. (2016) e Vale do Pinhão } \\
\text { (2018) }\end{array}$ \\
\hline Veículos Autônomos & $\begin{array}{c}\text { Pública / } \\
\text { Privada }\end{array}$ & MS & Benevolo et al. (2016) \\
\hline $\begin{array}{c}\text { Integração Temporal por } \\
\text { Bilhete Eletrônico }\end{array}$ & Pública & RC e RCT & Witiuk e von der Osten. (2018b) \\
\hline $\begin{array}{c}\text { Aplicativos de carpooling para } \\
\text { polos geradores de tráfego }\end{array}$ & Privada & RC e RCT & Benevolo et al. (2016) \\
\hline $\begin{array}{c}\text { Bicicletas compartilhadas } \\
\text { Sincronização semafórica }\end{array}$ & Privada & RP, RC, RB e RTC & Benevolo et al. (2016) \\
\hline $\begin{array}{c}\text { Sistema de orientação para } \\
\text { vagas de estacionamento }\end{array}$ & Infraestrutura & RP, RC e RCT & Benevolo et al. (2016) \\
\hline $\begin{array}{c}\text { Centro de operação e } \\
\text { controle de tráfego }\end{array}$ & Infraestrutura & RP, RC, MS, RB e & RCT \\
\hline $\begin{array}{c}\text { Sistemas de coleta de dados } \\
\text { de tráfego }\end{array}$ & Infraestrutura & $\begin{array}{c}\text { RP, RC, MS, RB e } \\
\text { RCT }\end{array}$ & Benevolo et al. (2016) \\
\hline
\end{tabular}

Fonte: Os autores

\section{CONCLUSÕES}

Este texto apresentou algumas das propostas de mobilidade que têm contribuído para a formação de um ecossistema inteligente nas cidades. Nesse sentido, é valorizada a herança deixada pelo planejamento no transporte público para a formação da classe criativa de Curitiba. No âmbito geral do estudo, o município apresentou bons resultados nas quatro sessões de análise. Todavia, sua divisão modal, que apresenta $46 \%$ de participação do automóvel individual, é tida como um empecilho para a formação de um ecossistema criativo.

Ao longo do trabalho, foram apresentados autores que corroboram com a noção da mobilidade como um importante fator para a formação de uma smart city. Na escala do Vale do Pinhão notabiliza-se, portanto, a necessidade das propostas do projeto voltadas à mobilidade e são sugeridas pelos autores outras iniciativas, no sentido de fomentar ainda mais 
o uso de sistemas inteligentes de transportes e da mobilidade ativa. Resulta disto o entendimento de que deve ser valorizada a mobilidade, como forma de integração dos espaços, e a infraestrutura de transporte, como o meio pelo qual ocorre o vínculo entre o usuário de transporte e a cidade.

Em suma, tendo em vista seu caráter multidisciplinar e de elevado grau de inovação, o projeto Vale do Pinhão é uma oportunidade singular para promover o encontro e a cooperação entre o setor público, setor privado e a universidade, na melhoria da mobilidade urbana visando a almejada condição de cidade inteligente.

\section{REFERÊNCIAS}

ABBOTT, Carl. Greater Portland: Urban life and landscape in the Pacific Northwest. University of Pennsylvania Press, 2001 p. 8-40.

BENEVOLO, Clara; DAMERI, Renata Paola; D'AURIA, Beatrice. Smart mobility in smart city. Empowering Organizations. Springer, Cham, 2016. p. 13-28.

BALULA, Luís. Espaço público e criatividade urbana: A dinâmica dos lugares em três bairros culturais. Cidades, Comunidades e Territórios, n. 20-21, 2010.

CARAGLIU, Andrea; DEL BO, Chiara; NIJKAMP, Peter. Smart cities in Europe. Journal of urban technology, v. 18, n. 2, p. 65-82, 2011.

COSTA, Pedro; SEIXAS, João; OLIVEIRA, Ana Roldão. Das Cidades Criativas à Criatividade Urbana? Espaço, Criatividade e Governança na Cidade Contemporânea. XV Encontro da APDR Redes e Desenvolvimento Regional, Cabo Verde, p. 6-11, 2009.

FERREIRA, Victor Moura Soares et al. A rede de cidades criativas da Unesco: uma perspectiva das cidades brasileiras. Goiânia: UFG, 2017.

GIFFINGER, RUdolf; GUDRUN, Haindlmaier. Smart cities ranking: an effective instrument for the positioning of the cities?. ACE: Architecture, City and Environment, v. 4, n. 12, p. 7-26, 2010.

IPPUC. Apresentação dos Resultados da Pesquisa Origem Destino. 2017. Disponível em: $<\mathrm{http}$ ://www.ippuc.org.br/mostrarpagina.php?pagina=536\&idioma=1 \&ampliar=n\%E3o $>$. Acesso em: 06 jan. 2019.

LARA, Alexander Prado et al. Projeto Florip@ 21: a construção de uma região inteligente na cidade de Florianópolis, Brasil. In: Proceedings of XV Latin Iberian-American Congress on Management of Technology, Porto. 2013. p. 1673-1691.

HUERTAS, Daniel Monteiro. Dinâmicas territoriais dos principais nodais sulistas do transporte rodoviário de carga. Raega-O Espaço Geográfico em Análise, v. 37, p. 160-189, 2016.

MIGUEZ, Paulo. Economia criativa: uma discussão preliminar. Teorias e políticas da cultura: visões multidisciplinares. Salvador: EDUFBA, p. 95-114, 2007.

NASCIMENTO, Patrícia Danielle Gomes do; DOMINGOS, Renata Cristina Navarro; LELLIS, Renata Facchini. Desenvolvimento do cluster de economia criativa na área central da cidade de São Paulo. São Paulo: FGV, 2015.

NUNES, André. Vale do Pinhão será o centro da Região Metropolitana em novo desenho urbano do Ippuc. Gazeta do Povo. 2017. Disponível em:

<https://www.gazetadopovo.com.br/haus/reacao-urbana/um-novo-tracado-para-curitibae-regiao/> Acesso em: 06 abr. 2019. 
PROCOPIUCK, Mario; FREDER, Schirlei. Políticas públicas de fomento à economia criativa: Curitiba e contexto nacional e internacional. Revista Brasileira de Planejamento e Desenvolvimento, v. 2, n. 2, p. 15-29, 2013.

SPECK, Jeff. Walkable city: how downtown can save America, one step at a time. $1^{\text {st }}$ ed. New York: North point press: Farrar, Straus and Giroux, 2013. 320 p.

MINISTÉRIO DOS TRANSPORTES, PORTOS E AVIAÇÃO CIVIL. Pesquisa de Satisfação do passageiro, Brasil, 2018. Disponível em: <http://transportes.gov.br/pesquisa-satisfacao.html> acesso em: 06 abr. 2019.

TRINDADE, Lucas Xavier; GÓES, Antônio Oscar Santos. O processo inovador através de um estudo de caso no Porto Digital-Recife: a tecnologia como vantagem competitiva. In: $X$ CONGRESSO NACIONAL DE EXCELÊNCIA EM GESTÃO, 2014, Rio de Janeiro. Anais. Rio de Janeiro: UFF, 2014.

WITIUK, Rafael Lopes; VON DER OSTEN Fabiana Bartalini. Estudo de viabilidade da carona como um modal de transporte atrativo para o campus Curitiba da PUCPR. I Simpósio de Transportes do Paraná. Anais. Curitiba. v. 1, n. 1. 2018

WITIUK, Rafael Lopes; VON DER OSTEN Fabiana Bartalini. A integração tarifária temporal em um contexto latino-americano. XX Congreso Latinoamericano de Transporte Público y Urbano (CLATPU). Anais. Medellín. v. 1, n. 1, p. 767-778. 2018

VALE DO PINHÃO. O que é o Vale do Pinhão. 2017. Disponovel em:

<http://www.valedopinhao.agenciacuritiba.com.br/institucional> Acesso em: 06 abr. 2019. 